\title{
Riociguat: an upcoming therapy in chronic thromboembolic pulmonary hypertension?
}

\author{
N.H. Kim
}

ABSTRACT: Although pulmonary endarterectomy remains the treatment of choice for patients with chronic thromboembolic pulmonary hypertension (CTEPH), not all patients will benefit from or receive this highly specialised surgery. Patients whose CTEPH is deemed inoperable by an experienced centre and patients with persistent pulmonary hypertension after surgery are candidates for trial of pulmonary arterial hypertension (PAH) specific pharmacotherapies. However, the currently available PAH specific pharmacotherapies have not demonstrated a clear benefit in either of these patient groups. Accordingly, PAH therapies remain off-label for use in CTEPH.

Riociguat (BAY 63-2521) is a stimulator of soluble guanylate cyclase, and may represent a novel agent in the treatment of select patients with CTEPH. Pre-clinical and human phase II studies with riociguat have reported promising results, and a multinational, randomised, controlled, doubleblinded phase III study is currently underway to investigate the effect of riociguat in patients with inoperable CTEPH and those with persistent or recurrent pulmonary hypertension following pulmonary endarterectomy.

KEYWORDS: Chronic thromboembolic pulmonary hypertension, haemodynamics, riociguat, soluble guanylate cyclase

hronic thromboembolic pulmonary hypertension (CTEPH) is an insidious, debilitating disease that often remains undiagnosed until it has reached an advanced stage [1]. Without treatment, the mean survival rate following diagnosis is $6.8 \mathrm{yrs}$ [2]. The treatment of choice for CTEPH is pulmonary endarterectomy (PEA), which can resolve symptoms and dramatically improve prognosis [3-5]. However, CTEPH is deemed inoperable in $20-40 \%$ of patients [6], and $10-20 \%$ of patients who undergo PEA have persistent or recurrent pulmonary hypertension following surgery $[7,8]$. Therefore, an unmet medical need for pharmacotherapies exists. This review will describe the use of pulmonary arterial hypertension (PAH) pharmacotherapies in CTEPH, discuss the implications of previous clinical study results, and summarise pre-clinical and clinical data for riociguat (BAY 63-2521); a new drug with a novel mode of action currently being investigated in phase III clinical studies as a treatment for CTEPH and PAH.

\section{PAH PHARMACOTHERAPIES IN CTEPH: RATIONALE}

$\mathrm{PAH}$ is characterised by an imbalance between endogenous vasodilators (nitric oxide and prostacyclin) and vasoconstrictors (endothelin), leading to pulmonary vasoconstriction and cell proliferation (vascular remodelling) [9]. There is evidence to suggest that a similar process may play a role in the development of CTEPH. As reviewed in detail elsewhere in this issue of the European Respiratory Review [1], small distal pulmonary vessels from patients with CTEPH show a proliferative phenotype (vascular remodelling and plexogenic lesions) similar to that observed in PAH [10]. In addition, nitric oxide signalling may be reduced in CTEPH; plasma levels of asymmetric dimethylarginine, an endogenous inhibitor of nitric oxide synthase, were found to be increased in patients with CTEPH compared with controls [11]. Thus, CTEPH and PAH may represent extremes of the same disease continuum [12] and PAH pharmacotherapies might, therefore, be expected to have a beneficial effect in certain patients with CTEPH who have a predominantly distal, PAH-like arteriopathy.

\section{WHEN MIGHT PHARMACOLOGICAL THERAPY BE APPROPRIATE?}

As yet, no drug has been approved for the treatment of CTEPH in the USA or Europe, and targeted pharmacotherapies for $\mathrm{PAH}$ are increasingly being used off-label for this indication.
CORRESPONDENCE

N.H. Kim

Division of Pulmonary and Critical

Care Medicine

University of California

San Diego

Mail Code 7381

9330 Campus Point Drive

La Jolla

CA 92037-1300

USA

E-mail: h33kim@ucsd.edu

Received:

Dec 012009

Accepted after revision:

Dec 212009

PROVENANCE

Publication of this peer-reviewed article was supported by Bayer Schering Pharma AG, Germany (principal sponsor, European Respiratory Review issue 115). 
For example, a retrospective chart analysis from the University of California (San Diego, CA, USA) found that the prevalence of $\mathrm{PAH}$ pharmacotherapies in patients referred for PEA increased from $20 \%$ in 2005 to $37 \%$ in 2007 [13]. Pre-operative treatment with PAH pharmacotherapies was associated with a delay in referral for PEA and had no effect on post-surgical outcome [13]. While this study does not support the widespread use of PAH pharmacotherapies to treat CTEPH prior to referral, they may be appropriate for certain patients after evaluation at a specialist centre. For example, pharmacotherapy may be considered if PEA is not possible or is unsuccessful. Persistent pulmonary hypertension following the removal of surgically accessible obstructions by PEA is likely to be due to residual distal arteriopathy and may, therefore, respond to pharmacological treatment. In patients with inoperable CTEPH (because of surgically inaccessible obstructions, significant distal arteriopathy or comorbidities that preclude surgery) pharmacotherapy may also be considered.

Pulmonary vascular resistance (PVR) remains an important preoperative measurement in the assessment of operative risk in CTEPH. Patients with CTEPH and a PVR of $>1,100 \mathrm{dyn} \cdot \mathrm{s} \cdot \mathrm{cm}^{-5}$ face higher peri-operative mortality and increased likelihood of persistent post-operative pulmonary hypertension [14]. Pharmacotherapy may help to reduce the degree of pulmonary hypertension in a subset of patients with severe CTEPH and thus serve as a bridge to surgery [6]. Some encouraging results have been obtained with epoprostenol, although responses were variable and need to be studied further [15, 16]. A small, randomised controlled study of bosentan $(n=25)$ showed haemodynamic and functional benefits when it was administered before surgery; however, a larger study is required to confirm whether this will translate into a reduction in mortality associated with PEA [17].

\section{CLINICAL TRIALS OF PAH PHARMACOLOGICAL THERAPIES IN CTEPH}

Although many uncontrolled studies have shown a potential beneficial effect of $\mathrm{PAH}$ pharmacotherapies in inoperable
CTEPH, only three randomised controlled trials have been performed [18], and none has led to regulatory approval in Europe or the USA (table 1). The 12-week Aerosolized Iloprost Randomized study enrolled 203 patients with pulmonary hypertension, including 57 patients with inoperable CTEPH. Compared with placebo, iloprost was associated with haemodynamic and functional improvements, with the primary pulmonary hypertension group benefiting more than the group of patients with nonprimary pulmonary hypertension, including those with CTEPH. However, a detailed subgroup analysis of patients with CTEPH in this study was not reported [19]. A small, 12-week clinical study of sildenafil in 19 patients with inoperable CTEPH failed to meet its primary end-point (change in 6-min walking distance (6MWD)) [20] and the pivotal phase III study of bosentan (Bosentan Effects in iNopErable Forms of chronIc Thromboembolic pulmonary hypertension; BENEFIT $)(n=157)$ also failed to show an improvement in 6MWD after 16 weeks of treatment, despite there being a significant decrease in PVR compared with placebo [21].

The failure of previous randomised controlled trials in CTEPH could be due to several factors other than lack of efficacy. For instance, the choice of end-point may have been inappropriate; the use of 6MWD, although used in virtually all previous studies of PAH specific therapy as an indicator of efficacy [22], may be insensitive in the context of clinical trials in CTEPH. For example, the average age of patients with CTEPH in the BENEFIT study was 63 yrs; the authors noted that this was rather high compared with typical PAH study populations, and suggested that additional age-related factors may have contributed to the impairment of exercise capacity in their study population [21]. The duration of the studies may also not have been long enough to detect an effect on 6MWD. In a retrospective study of 102 patients who underwent PEA, $6 \mathrm{MWD}$ improved gradually over the course of $1 \mathrm{yr}$ following surgery, despite an immediate improvement in pulmonary haemodynamic parameters [23]. Finally, all three studies

\section{TABLE 1 \\ Randomised controlled trials of pulmonary arterial hypertension pharmacotherapies in chronic thromboembolic} pulmonary hypertension (CTEPH)

\begin{tabular}{|c|c|c|c|}
\hline Target pathway & Prostacyclin & Nitric oxide & Endothelin \\
\hline Subjects $n$ & 57 CTEPH $(203 \text { total) })^{\#}$ & 19 CTEPH & 157 CTEPH \\
\hline Duration of study & 12 weeks & 12 weeks & 16 weeks \\
\hline Primary end-point & $6 \mathrm{MWD}+\mathrm{NYHA}$ functional class & $6 \mathrm{MWD}$ & 6MWD or PVR \\
\hline $\begin{array}{l}\text { Change in PVR versus placebo } \\
\text { dyn } \cdot \mathbf{s} \cdot \mathbf{c m}^{-5}\end{array}$ & $\begin{array}{l}-105^{\star} \text { (pre-inhalation) } \\
-335^{5} \text { (post-inhalation) }\end{array}$ & $-197^{\star}$ & $-176^{\star}$ \\
\hline Change in 6MWD versus placebo $\mathrm{m}$ & $+36.4^{*}(\mathrm{PPH}=58.8 ; \mathrm{nPPH}=12)$ & +17.5 & +2.2 \\
\hline
\end{tabular}

PVR: pulmonary vascular resistance; 6MWD: 6-min walking distance; NYHA: New York Heart Association; PPH: primary pulmonary hypertension; nPPH: nonPPH (including CTEPH and pulmonary hypertension associated with anorexigen use or scleroderma). ${ }^{*}$ : separate analyses for the CTEPH subgroup were not presented but type of pulmonary hypertension had no significant effect on the response to treatment; ${ }^{\imath}$ : independent co-primary end-points; ${ }^{\text {s: }}$ significance versus placebo not assessed. *: $p<0.05$. 
enrolled patients with inoperable CTEPH. This is a heterogeneous group that is defined subjectively. The amalgam of "inoperable" CTEPH incorporates both predominantly distal arteriopathy and predominantly proximal CTEPH phenotypes. Although patients with the former pattern may be expected to benefit from pharmacotherapy, it is unclear whether those showing the latter pattern would show a clinical response.

\section{RIOCIGUAT: A NEW MODE OF ACTION TARGETING THE NITRIC OXIDE PATHWAY}

Riociguat is a first-in-class, orally available drug that is being developed as a treatment for CTEPH and PAH. It stimulates soluble guanylate cyclase (sGC), a key component of the nitric oxide signalling pathway that binds nitric oxide and increases synthesis of the second messenger cyclic guanosine monophosphate (fig. 1) [24, 25]. Riociguat has a dual mode of action; it increases the sensitivity of sGC to endogenous nitric oxide by stabilising the binding of nitric oxide to the prosthetic haem group on sGC, and it can also increase sGC activity independently of nitric oxide [27]. The nitric oxide independent action of riociguat distinguishes it from phosphodiesterase-5 inhibitors, which require nitric oxide to function and may have limited effect in the presence of very low nitric oxide levels [28]. The therapeutic potential of riociguat was first demonstrated in mice and rats with established pulmonary hypertension; treatment with riociguat led to partial reversal of pulmonary hypertension, vascular remodelling and cardiac hypertrophy in these models [27].

\section{CLINICAL DEVELOPMENT OF RIOCIGUAT}

Oral riociguat was rapidly absorbed and had a favourable safety profile in healthy volunteers [29] and patients with pulmonary hypertension [30]. In a proof of concept study in 19 patients with pulmonary hypertension, the haemodynamic response to riociguat correlated with its plasma concentration

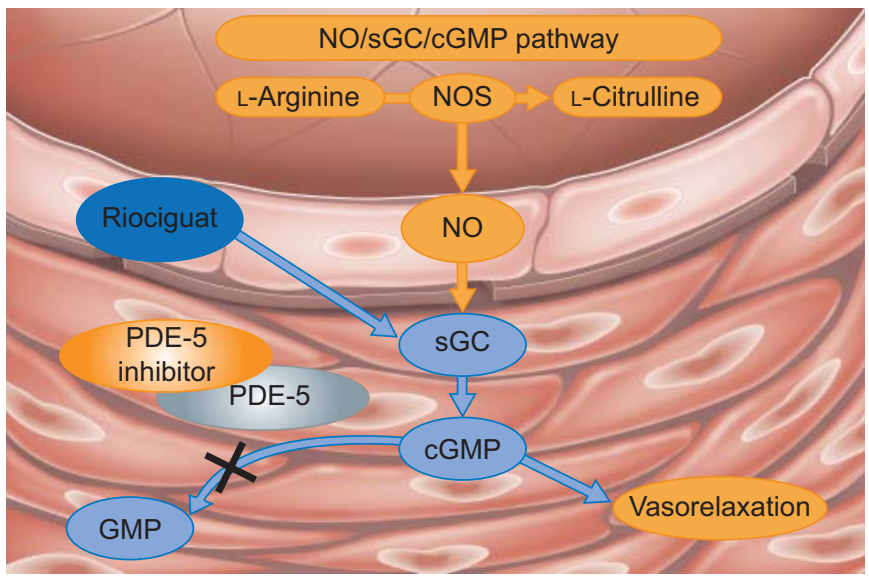

FIGURE 1. Therapeutic approaches targeting the nitric oxide (NO) signalling pathway in pulmonary hypertension. Riociguat stimulates soluble guanylate cyclase (sGC) with a dual mode of action. When sufficient NO is present, riociguat acts in synergy with NO, but it can also stimulate sGC directly when NO is absent or scarce. Phosphodiesterase (PDE)-5 inhibitors act further downstream in the pathway, preventing degradation of cyclic guanosine monophosphate (cGMP). Thus, their efficacy depends on the presence of an intact NO/sGC/cGMP signalling pathway. NOS: NO synthase; GMP: guanosine monophosphate. Adapted from [26] with permission from the publisher. and riociguat significantly increased the cardiac index in patients with CTEPH, as well as in patients with PAH (fig. 2) [30]. A subsequent 12-week, phase II study of riociguat in 72 patients with CTEPH or PAH confirmed these promising haemodynamic results. Moreover, this first assessment of the effects of riociguat on exercise capacity showed very encouraging improvements from baseline in 6MWD in both patient subgroups (ClinicalTrials.gov identifier NCT00454558) [31]. Phase III studies in patients with CTEPH (CHEST; ClinicalTrials.gov identifier NCT00855465) and PAH (PATENT; ClinicalTrials.gov identifier NCT00810693) are now underway.

\section{CONCLUSIONS}

PEA is widely accepted as the treatment of choice for CTEPH, but it is not suitable for all patients, and a medical need for pharmacotherapy remains. Patients whose CTEPH is deemed inoperable by an experienced centre and patients with persistent post-PEA pulmonary hypertension are candidates for a trial of $\mathrm{PAH}$ pharmacotherapies. $\mathrm{PAH}$ specific pharmacotherapies may also be useful as a bridge to surgery in select patients with severe CTEPH. The decision for a trial of medical therapy in lieu of PEA, or bridging therapy prior to PEA should only be made in consultation with an experienced PEA centre.

Riociguat is an orally available, first-in-class drug that has shown promising results in open-label phase II studies of patients with CTEPH or PAH. The therapeutic potential of riociguat in $\mathrm{CTEPH}$ and $\mathrm{PAH}$ is now being investigated in phase III randomised controlled trials. To date, no randomised controlled clinical study has provided conclusive evidence to support the use of PAH pharmacotherapies in CTEPH. Moreover, introduction of such targeted agents should not delay patient referral to an expert centre for assessment of suitability for surgical intervention.

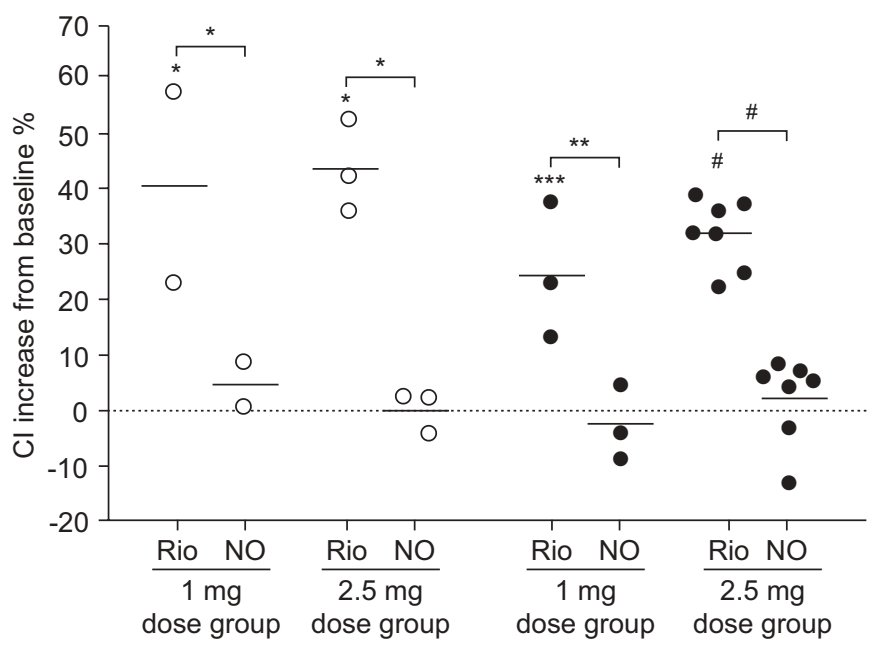

FIGURE 2. Cardiac index $(\mathrm{Cl})$ changes from baseline following single oral doses of riociguat (Rio) compared with inhaled nitric oxide (NO) in patients with chronic thromboembolic pulmonary hypertension $(\bigcirc)$ and pulmonary arterial hypertension $(\bullet)$. Horizontal lines indicate point estimates (least-squares means) Statistical significance was measured by the F statistic. ${ }^{*}: p<0.05$; ${ }^{*}: p<0.01$; *** $p<0.001 ;{ }^{*}: p<0.0001$. Reproduced from [30] with permission from the publisher 


\section{CLINICAL TRIALS}

This study is registered at ClinialTrials.gov identifier number NCT 00454558 .

\section{STATEMENT OF INTEREST}

N.H. Kim has received speaker honoraria from Actelion, Gilead and Bayer Schering Pharma AG, and has served on a steering committee for Bayer Schering Pharma AG.

\section{ACKNOWLEDGEMENTS}

Medical writing support was provided by C. Mulligan (Oxford PharmaGenesis Ltd, Oxford, UK) on behalf of Bayer Schering Pharma AG (Berlin, Germany). This article is based on a presentation given at a symposium supported by Bayer Schering Pharma AG at the 2009 European Society of Cardiology meeting in Barcelona, Spain.

\section{REFERENCES}

1 Humbert M. Pulmonary arterial hypertension and chronic thromboembolic pulmonary hypertension: pathophysiology. Eur Respir Rev 2010; 19: 59-63.

2 Kunieda T, Nakanishi N, Satoh T, et al. Prognoses of primary pulmonary hypertension and chronic major vessel thromboembolic pulmonary hypertension determined from cumulative survival curves. Intern Med 1999; 38: 543-546.

3 Archibald CJ, Auger WR, Fedullo PF, et al. Long-term outcome after pulmonary thromboendarterectomy. Am J Respir Crit Care Med 1999; 160: 523-528.

4 Galie N, Hoeper MM, Humbert M, et al. Guidelines for the diagnosis and treatment of pulmonary hypertension: The Task Force for the Diagnosis and Treatment of Pulmonary Hypertension of the European Society of Cardiology (ESC) and the European Respiratory Society (ERS), endorsed by the International Society of Heart and Lung Transplantation (ISHLT). Eur Heart J 2009; 30: 2493-2537.

5 McLaughlin VV, Archer SL, Badesch DB, et al. ACCF/AHA 2009 expert consensus document on pulmonary hypertension a report of the American College of Cardiology Foundation Task Force on Expert Consensus Documents and the American Heart Association developed in collaboration with the American College of Chest Physicians; American Thoracic Society, Inc.; and the Pulmonary Hypertension Association. J Am Coll Cardiol 2009; 53: 1573-619.

6 Mayer E. Surgical and post-operative treatment of chronic thromboembolic pulmonary hypertension. Eur Respir Rev 2010; 19: 64-67.

7 Bonderman D, Skoro-Sajer N, Jakowitsch J, et al. Predictors of outcome in chronic thromboembolic pulmonary hypertension. Circulation 2007; 115: 2153-2158.

8 Galie N, Kim NH. Pulmonary microvascular disease in chronic thromboembolic pulmonary hypertension. Proc Am Thorac Soc 2006; 3: 571-576.

9 McLaughlin VV, McGoon MD. Pulmonary arterial hypertension. Circulation 2006; 114: 1417-1431.

10 Moser KM, Bloor CM. Pulmonary vascular lesions occurring in patients with chronic major vessel thromboembolic pulmonary hypertension. Chest 1993; 103: 685-692.

11 Skoro-Sajer N, Mittermayer F, Panzenboeck A, et al. Asymmetric dimethylarginine is increased in chronic thromboembolic pulmonary hypertension. Am J Respir Crit Care Med 2007; 176: 1154-1160.

12 Peacock A, Simonneau G, Rubin L. Controversies, uncertainties and future research on the treatment of chronic thromboembolic pulmonary hypertension. Proc Am Thorac Soc 2006; 3: 608-614.
13 Jensen KW, Kerr KM, Fedullo PF, et al. Pulmonary hypertensive medical therapy in chronic thromboembolic pulmonary hypertension before pulmonary thromboendarterectomy. Circulation 2009; 120: $1248-1254$

14 Hartz RS, Byrne JG, Levitsky S, et al. Predictors of mortality in pulmonary thromboendarterectomy. Ann Thorac Surg 1996; 62: 1255-1259.

15 Nagaya N, Sasaki N, Ando M, et al. Prostacyclin therapy before pulmonary thromboendarterectomy in patients with chronic thromboembolic pulmonary hypertension. Chest 2003; 123: 338-343.

16 Bresser P, Fedullo PF, Auger WR, et al. Continuous intravenous epoprostenol for chronic thromboembolic pulmonary hypertension. Eur Respir J 2004; 23: 595-600.

17 Reesink HJ, Surie S, Kloek JJ, et al. Bosentan as a bridge to pulmonary endarterectomy for chronic thromboembolic pulmonary hypertension. J Thorac Cardiovasc Surg 2010; 139: 85-91.

18 Lang IM. Managing chronic thromboembolic pulmonary hypertension: pharmacological treatment options. Eur Respir Rev 2009; 18: $24-28$

19 Olschewski H, Simonneau G, Galie N, et al. Inhaled iloprost for severe pulmonary hypertension. N Engl J Med 2002; 347: 322-329.

20 Suntharalingam J, Treacy CM, Doughty NJ, et al. Long term use of sildenafil in inoperable chronic thromboembolic pulmonary hypertension. Chest 2008; 134: 229-236.

21 Jais X, D'Armini AM, Jansa P, et al. Bosentan for treatment of inoperable chronic thromboembolic pulmonary hypertension: BENEFiT (Bosentan Effects in iNopErable Forms of chronIc Thromboembolic pulmonary hypertension), a randomized, placebo-controlled trial. J Am Coll Cardiol 2008; 52: 2127-2134.

22 Rich S. The current treatment of pulmonary arterial hypertension: time to redefine success. Chest 2006; 130: 1198-1202.

23 Matsuda H, Ogino H, Minatoya $\mathrm{K}$, et al. Long-term recovery of exercise ability after pulmonary endarterectomy for chronic thromboembolic pulmonary hypertension. Ann Thorac Surg 2006; 82: 1338-1343.

24 Stasch JP, Hobbs AJ. NO-independent, haem-dependent soluble guanylate cyclase stimulators. Handb Exp Pharmacol 2009; 191: 277-308.

25 Ghofrani HA, Grimminger F. Soluble guanylate cyclase stimulation: an emerging option in pulmonary hypertension therapy. Eur Respir Rev 2009; 18: 35-41.

26 Ghofrani A, Grimminger F. Modulating cGMP to treat lung diseases. In: Schmidt HHHW, Hofmann F, Stasch JP, eds. cGMP: Generators, Effectors and Therapeutic Implications. SpringerVerlag, Berlin, 2009; pp. 469-483.

27 Schermuly R, Stasch JP, Pullamsetti SS, et al. Expression and function of soluble guanylate cyclase in pulmonary arterial hypertension. Eur Respir J 2008; 32: 881-891.

28 Evgenov OV, Pacher P, Schmidt PM, et al. NO-independent stimulators and activators of soluble guanylate cyclase: discovery and therapeutic potential. Nat Rev Drug Discov 2006; 5: 755-768.

29 Frey R, Mück W, Unger S, et al. Single-dose pharmacokinetics, tolerability and safety of the soluble guanylate cyclase stimulator BAY 63-2521; an ascending-dose study in healthy male volunteers. J Clin Pharmacol 2008; 48: 926-934.

30 Grimminger F, Weimann G, Frey R, et al. First acute haemodynamic study of soluble guanylate cyclase stimulator riociguat in pulmonary hypertension. Eur Respir J 2009; 33: 785-792.

31 Ghofrani HA, Hoeper MM, Hoeffken G, et al. Riociguat dose titration in patients with chronic thromboembolic pulmonary hypertension (CTEPH) or pulmonary arterial hypertension (PAH). Am J Respir Crit Care Med 2009; 179: A3337. 